\title{
Sonografiekurse nach den Richtlinien der ÖGUM
}

\section{Detaillierte Infos im Kurs-Kalender unter: www.oegum.at/ausbildung/kurskalender.html}

- 13. - 14.01.2018 Wien: Abdomensonografie Grundkurs nach ÖGUM inkl. Notfallsonografie

Leitung \& Auskunft: OA Dr. Aiginger, www.ultraschallkurs.at

- 04. - 07.03.2018 Gaschurn: Abdomen Refresherkurs

Leitung \& Auskunft: Prof. Dr. Schuler, Prof. Dr. Mathis,

www.ultraschallkursegaschurn.de

- 08. - 10.03.2018 Hohenems: Abdomensonografie Grundkurs Leitung \& Auskunft: OA Dr. Gehmacher, sekretariat.interne@vlkh.net
- 11. - 12.03.2018 Wien: Grundkurs Abdomenultraschall nach OEGUM Leitung \& Auskunft: OA Dr. Ybinger, www.ultraschallkurse-wien.at

- 12. - 14.04.2018 Wien: Ultraschall Grundkurs Abdomen

Univ. Prof. Dr. Mostbeck und Univ. Prof. Dr. Dock,

www.wienerultraschallseminare.at

- 04. - 06.10.2018 Hohenems: Abdomensonografie Grundkurs Leitung \& Auskunft: OA Dr. Gehmacher, sekretariat.interne@vlkh.net
- 08. - 11.11.2018 Hohenems: Abdomensonografie Aufbauund Abschlusskurs

Leitung \& Auskunft: OA Dr. Gehmacher, sekretariat.interne@vlkh.net

- 07. - 08.11.2018 Baden: Ultraschall Kurs Abdomen inkl. Gastrointestinaltrakt

Leitung \& Auskunft: Univ. Prof. Dr. Gritzmann, norbert.gritzmann@gmail.com, www.sonoseminare.com 


\section{Bewegungsapparat}

- 14. - 16.12.2017 Wien: 8th Interdisciplinary Musculoskeletal Ultrasound Course Vienna

Leitung \& Auskunft: Dr. Platzgummer, Prof. Dr. Weidekamm, us-course-vienna@meduniwien.ac.at

- 19.01.2018 Baden: Ultraschall gezielte Regionalanästhesie und -Therapie Leitung \& Auskunft: Univ. Prof. Dr. Gritzmann, norbert.gritzmann@gmail.com, www.sonoseminare.com

- 20. - 21.04.2018 Baden: Ultraschallkurs Gelenke, Weichteile und Nerven Leitung \& Auskunft: Univ. Prof. Dr. Gritzmann, norbert.gritzmann@gmail.com, www.sonoseminare.com

\section{Gefäße}

- 03. - 04.03.2018 Graz: Fortgeschrittenenkurs/Refresherkurs (Abschlusskurs) Doppler und Farbduplex-Sonografie der extra- und intracraniellen Hirnarterien Leitung \& Auskunft: Univ. Prof. Dr. Niederkorn,office@niederkorn.at oder k.niederkorn@niederkorn.at, T: 0043

(0)316 318 208, F: 0043 (0)316 $318208-24$

- 03. - 04.03.2018 Innsbruck: Dopplerund Duplexsonografie der hirnversorgenden Arterien

Leitung \& Auskunft: Dr. Schmidauer, christine.span@tirol-kliniken.at

- 21. - 22.04.2018 Graz: EinführungsAufbaukurs (Grundkurs) Doppler und Farbduplex-Sonografie der extra- und intracraniellen Hirnarterien

Leitung \& Auskunft: Univ. Prof. Dr. Niederkorn,office@niederkorn.at oder k.niederkorn@niederkorn.at

- 09. - 10.11.2018 Baden: Farbdopplersonografiekurs „Periphere Gefäße und Halsgefäße“

Leitung \& Auskunft: Univ. Prof. Dr. Gritzmann, norbert.gritzmann@gmail.com, www.sonoseminare.com
Gynäkologie

- 21.09. - 22.09.2018 Schloss Seggau/ Steiermark: Ultraschall Update5 Seggau

Leitung \& Auskunft: Univ.-Prof. Dr. Häusler, Univ.-Prof. Dr. Klaritsch, OA Dr. Csapo, www.ultraschallupdate.at

\section{Notfallsonografie}

- 18.01.2018 Baden: Ultraschallkurs für Intensiv/Notfallmediziner inkl. EFAST Leitung \& Auskunft: Univ. Prof. Dr. Gritzmann, norbert.gritzmann@gmail.com, www.sonoseminare.com

- 25.02.2018 Wien: EFAST - Notfallsonografie (nach ÖGUM Richtlinie) Leitung \& Auskunft: OA Dr. Ybinger, www.ultraschallkurse-wien.at

- 26. - 27.05.2018 Feldkirch: 6. Feldkircher Workshop „Anästhesie-fokussierte Sonografie“"

Leitung \& Auskunft: Dr. Gorsewski, Dr. Reithmeier, anaesthesie.sekretariat@lkhf.at

- 05.10.2018 Baden: Notfallsonografiekurs/EFAST Kurs

Leitung \& Auskunft: Univ. Prof. Dr. Gritzmann, norbert.gritzmann@gmail.com, www.sonoseminare.com

\section{Pädiatrie/Bewegungsapparat}

- 20. - 22.01.2018 Wien: Intensivkurs Hüftsonografie

Leitung \& Auskunft: OÄ Dr. Pospischill, sonokurs@oss.at

- 22.01.2018 Wien: Abschlusskurs Hüftsonografie Leitung \& Auskunft: OÄ Dr. Pospischill, sonokurs@oss.at

- 28.02. - 01.03.2018 Stolzalpe: Sonografie der Säuglingshüfte nach Graf Leitung \& Auskunft: OA Dr. Spieß, PD Dr. Tschauner, orthopaedie@LKH-Stolzalpe.at

- 20. -21.06.2018 Stolzalpe: Sonografie der Säuglingshüfte nach Graf Leitung \& Auskunft: OA Dr. Spieß, PD Dr. Tschauner, orthopaedie@LKH-Stolzalpe.at
- 19. - 20.09.2018 Stolzalpe: Sonografie der Säuglingshüfte nach Graf Leitung \& Auskunft: OA Dr. Spieß, PD Dr. Tschauner, orthopaedie@LKH-Stolzalpe.at

- 21. - 22.11.2018 Stolzalpe: Sonografie der Säuglingshüfte nach Graf Leitung \& Auskunft: OA Dr. Spieß, PD Dr. Tschauner, orthopaedie@LKH-Stolzalpe.at

\section{Interdisziplinär}

- 15.12.2017 Wien: Interdisziplinäre Ultraschall-Fortbildung Leitung \& Auskunft: Ass. Prof. Dr. Brichta, kostenfrei für alle Ärzte zugänglich

- 06. - 09.09.2018 Poznan/Polen: EUROSON 2018/30th Euroson Congress of the EFSUMB

www.euroson2018poznan.pl/en/

Kopf/Hals

- 19.04.2018 Baden: Ultraschallkurs Schilddrüse, Halsweichteile und Halsgefäße

Leitung \& Auskunft: Univ. Prof. Dr. Gritzmann, norbert.gritzmann@gmail.com, www.sonoseminare.com

- 02.05.2018 Sorrent/Italien: Grundkurs Schilddrüse

Leitung \& Auskunft: Doz. Dr. Zettinig, office@ce-management.com

- 03.05.2018 Sorrent/Italien: Aufbaukurse Schilddrüse

Leitung \& Auskunft: Doz. Dr. Zettinig, office@ce-management.com

Thorax

- 08. - 10.03.2018 Gaschurn: LungenPleurasono Kurs Leitung \& Auskunft: Prof. Dr. Schuler, Prof. Dr. Mathis, www.ultraschallkursegaschurn.de 